\title{
A Development of LBS by Using Beacon BLE Technology in Healthcare
}

\author{
Hadi Mesbah ${ }^{1}$, Omid Mahdi Ebadati E..$^{*}$ \\ \{std_h.mesbah@khu.ac.ir ${ }^{1}$, ebadati@khu.ac.ir $\left.{ }^{2}\right\}$ \\ ${ }^{1}$ Department of Information Technology Management, Kharazmi University, Tehran, Iran, ${ }^{2}$ Department of \\ Mathematics and Computer Science, Kharazmi University, Tehran, Iran
}

\begin{abstract}
Wireless sensor networks are becoming popular in health-care systems day by day with the rapid growth of new technologies and application, including Location-based aware systems. Following the comprehensive health-care reforms in past years, it motivates researchers to improve approachability to health-care specially in hospitals and health centers. The main purpose of this research is to develop a model for using of Location-based systems through iBeacon and Bluetooth Low Energy technologies in health-care. The proposed model is developed for hospitals as one of the active places, where time is critical. One of the major problems in the hospital environment is often the waiting time, procedures and finding appropriate departments, which are because of large population and the size of a hospital. To endeavor this dilemma, we developed a model that easily can help for the centers and places to visited by patients through their smartphone devices and examine it in a real indoor environment. The results show that, the proposed model can significantly improve hospital information management and patient guidance, and keep them up-to-date and provide detailed patient database, patient's information and location to keep them track of doctor's appointment, and receiving a medication. With using different sensors, BLS, iBeacon and BLE, we can align patient, doctor, nurse, and pharmacy vendor to the specific purpose of satisfying patient's needs.
\end{abstract}

Keywords: Wireless sensor networks, BLE (Bluetooth Low Energy) Technology, Beacon Technology, Hospital Services, LBS (Location Based Services)

\section{Introduction}

Nowadays, mobile technology offers solutions to a wide range of daily problems. Movable and related technologies to mobile technologies such as NFC, GPS, WiFi and Beacons are evolving and becoming an integral part of daily lives.

A new technology mostly refers to apprehensions of cost, interoperability, and compatibility. These issues formerly made delay for extensive adoption of technologies, like, wireless in medical applications [1].

In aforementioned researches, inadequate consideration has been given to a monitoring of health, eventually during a rescue mission [2]. To connect this gap, a research by [3] is proposed

* Corresponding Author 
that Wearable Wireless Sensor Networks (WWSNs) that could perform a significant role to improve current Public-Safety Network (PSN).

The hospital is one of the most important centers with long waiting time and receiving timely services for patients. In addition, finding different parts of the hospital are confusing because of the overpopulation and large hospital size. Other issues that cause patients' frustration in hospitals are the lack of responsiveness of admission units and absence of up-to-date information on bulletin boards and communication systems [4].

Today, these problems can be solved with a smartphone. Imagine that one enters the hospital and a welcome message appears on his phone upon arrival, then by entering the main area, he receives a message to follow the path to enter the unit. After crossing the path, he takes the necessary steps with the receptionist to take turns and make the payments. Now there is a message on the waiting time and the path to take to achieve the doctor's office in the hospital. Right now, he is at the doctor's office with the least amount of time wasted, and his medical record is complete and available to the doctor online. On the other hand, without holding the physical copy of the prescription he could refer to the pharmacy and receive his medicine [5].

These are possible by installing a limited number of small devices called the Beacon in the hospital environment. Wireless technology is leveraging a ready-built infrastructure for data transmission. Trends' analysis designated by this data can assist doctors to manage diseases like, diabetes.

In this research, we implement BLE iBeacon in a mid-size of hospital to gather data and generate significant information to guide patients, physicians and hospital manager. The proposed model reduced patients waiting time that lead to improve hospital performance.

\section{Literature Review}

One of the major applications of wireless systems is healthcare that possessed vital issues. Another requirement, which is mattered for developing countries is to have a low-cost and reliable network with effective protocols [6]. Beacons are based on Bluetooth Low Energy (BLE), which is a medium ranged transmission standard targeting for inexpensive devices with long life batteries [7, 8]. Beacon technology is a protocol first introduced by APPLE at the Apple Worldwide Developers International Conference 2013. This system has a very small hardware to provide information and location services, which transfers information to other devices, including a smartphone unilaterally. This device enables the transmission of information by Bluetooth low energy radio signals without prior acceptance (transmitter and receiver pairing). In simple words, this technique is something like GPS technology. Accordingly, the advantage of this device over GPS is the location in the indoors environments that implemented as a patent [9]. There is a new method to use a BLE to provide a GPS and indoor locating service without using the cellular network data which is simultaneous locating system based only on the Bluetooth low energy (BLE) technology to support collaborative communications by uniting the broadcast and mesh topology options to spread the applicability of beacon solutions [10].

There is another research that introduces a model for wearable sensors, and the focuses of researchers are in the real-time communication and data transmission of vital data of patients; those 
are in the critical situation [11]. The authors worked on the low-cost routing algorithm to reach realtime data transmission in the wireless body sensor network.

BLE can play a big role in health industry environment and acts as a CenterPoint of interconnection for all related monitoring apps and services. Figure 1 shows the data flow between BLE sensors, patients, machines and medical team. Moreover, the figure demonstrates the connection of data storage in local servers and remote healthcare centers for further use.

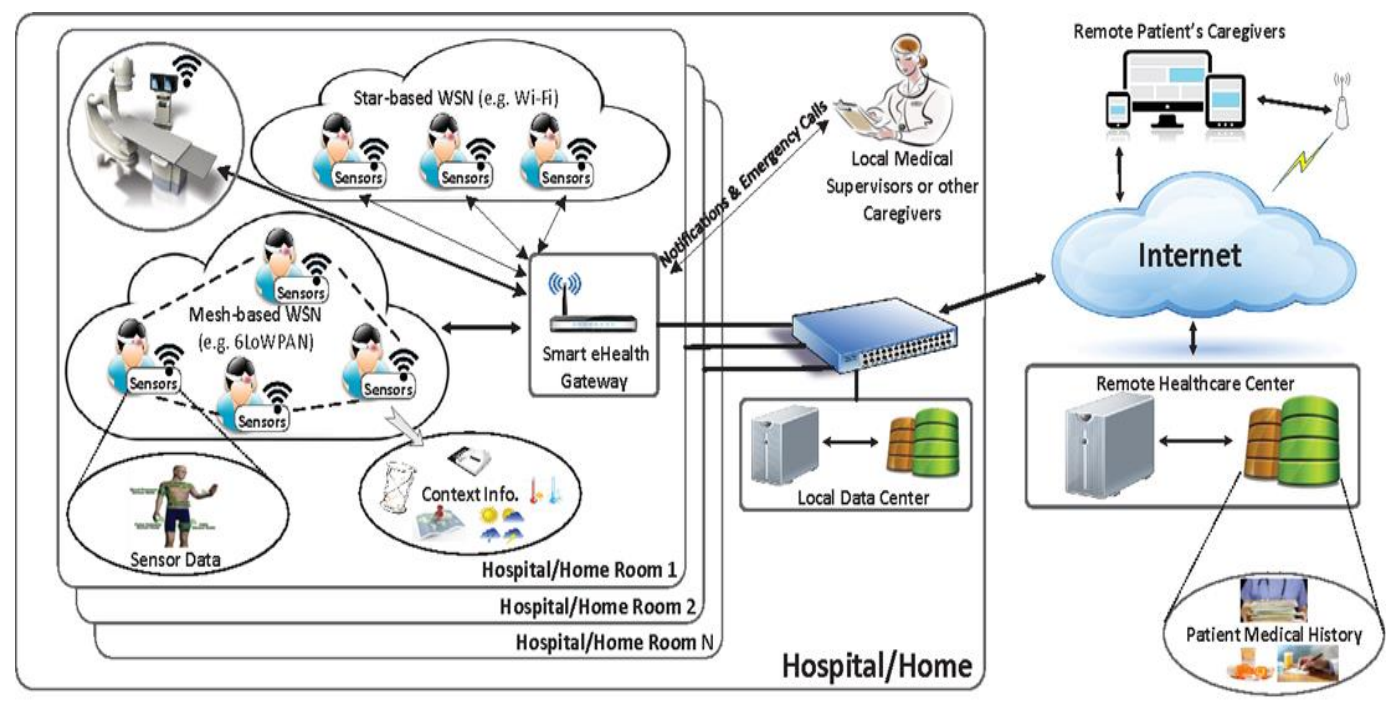

Fig. 1. Data flow between BLE sensors, patients and medical team [12]

The goal of this system is using the Bluetooth proximity sensor, the unique identifier received by different users, and the unique pre-programmed information of each user are transmitted to their smartphone via Bluetooth's port. Wearable sensor-based healthcare systems have drawn a lot of attention from the scientific community and the industry during the past several years. As the healthcare cost is growing, and the population is aging, unremarkable, low-cost and precise healthcare systems will possibly transform the healthcare forthcoming [13]. With this technique, it is possible to send comprehensive information to smart devices depending on the physical location and specific circumstances of each user. Location-based technologies are fully in line with the advancement of smartphones. Therefore, it can be concluded that this technique can make a valuable contribution to the medical industry, museums, shops, tourism industry, and most importantly to the management of information resources. Figure 2 presents the design and infrastructure that are needed to implement and use the BLW based solutions in hospitals and how patients and doctors can benefit of its implementation. 


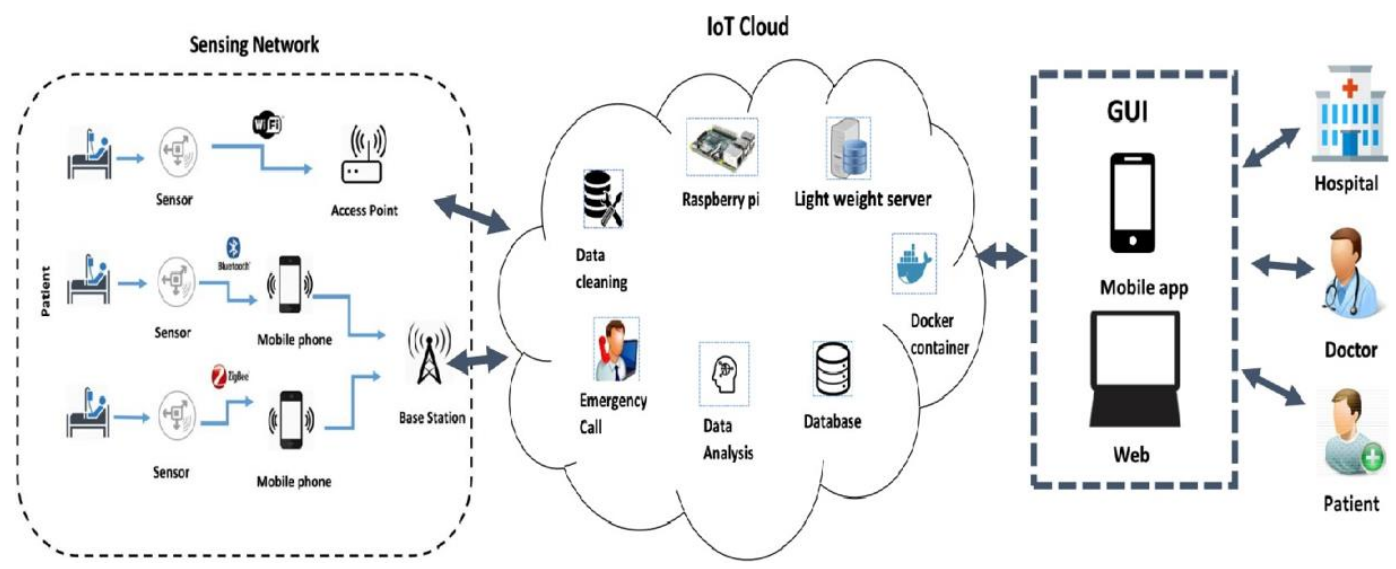

Fig. 2. Required modules and infrastructure in healthcare system [14]

The data-transfer platform in this technique is BLE. As its name implies, similar to its classical prototype, this technology is a personal wireless network for transmitting data over short distances but with far less power consumption. The power consumption of this network is so low that a simple button cell can easily last up to three years. This technique is suitable for transferring small data over short distances [15]. This technology transmits information to all the surrounding devices by releasing waves through the beacons [16]. Accordingly, it can be said that the information is being broadcasted by Beacons, and a device near the Beacons broadcast that information again. Depending on the device settings, these signals can be broadcasted between 100 and 200 meters. This difference depends on the settings set for the device. Figure 3 showing the detailed sensor network for in room and medical equipment's network.

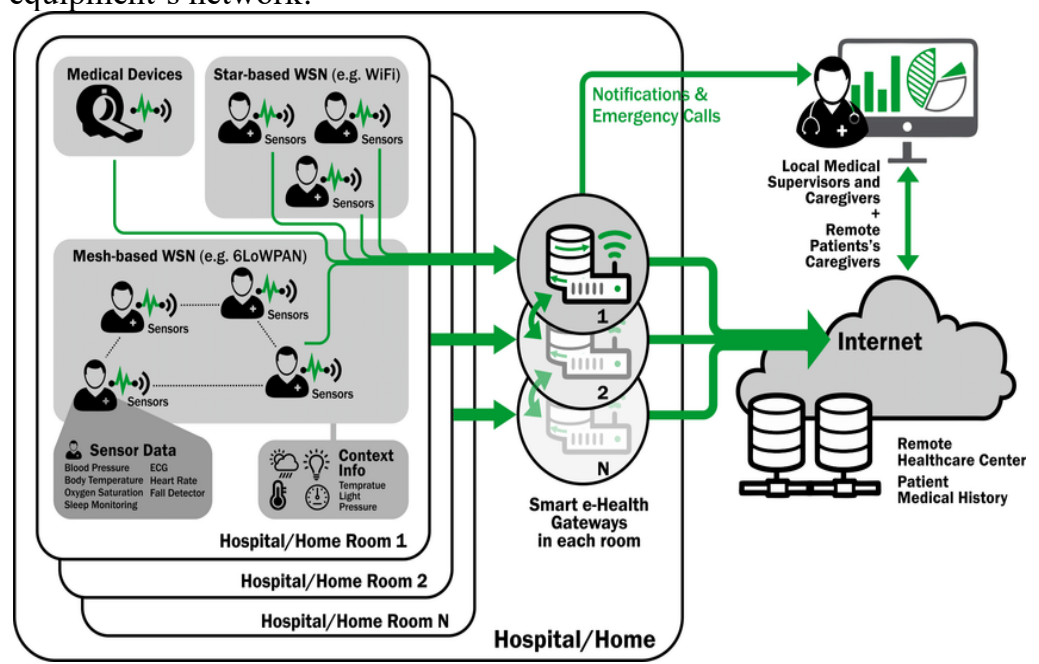

Fig. 3. How sensors send data to local, internet and remote locations [12] 
Variables used to change the signal-to-user distance of a Beacon device include:

- RSSI (Received Signal Strength Indicator)

- TX Power

Figure 4 shows the sample of APP GUI installed on cell phone to locate and be guided to the requested location by BLW sensors.
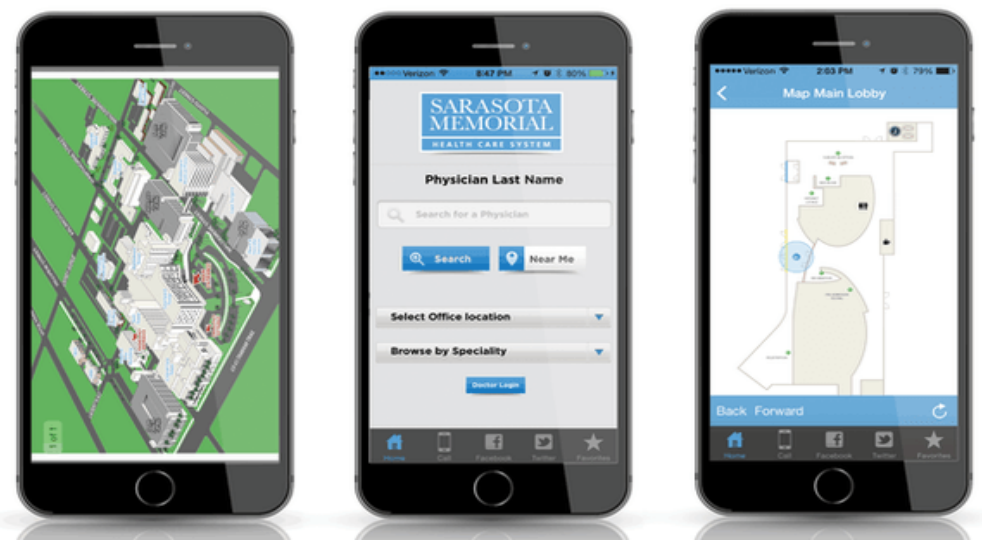

Fig. 4. Communication Model [16]

Figure 5 illustrates the solution based on the aforementioned articles, and layers required to implement this technology. Netherlands Department of Cardiology presented by the Leiden University and Medical Center (LUMC), designed the platform using IoT platforms with Beacon technology to treat patients with acute infectious disease. Therefore, the Sensor Base Tracking System was considered.

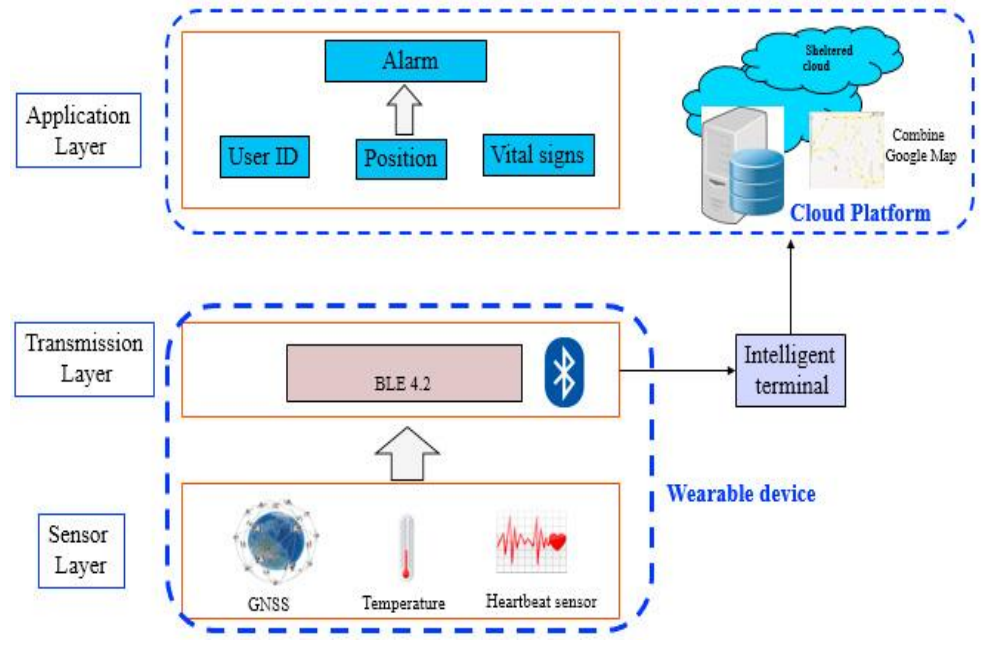

Fig. 5. Layers required to design a Beacon based solutions [10] 


\section{Research Method}

Based on the conducted studies, reviews and statistical data, the purpose of this study is to analyze the mentioned technology. From this point, a consensus is reached on the subject of the related services through the Location Base Services (LBS). The proposed method is based on LBS application in the field of treatment based on the conducted studies. Initially, the need in the field of treatment is examined, and then, by observing executive examples around the world, a conclusion on the use of this technology in the hospital setting is achieved.

\subsection{Communication via Bluetooth Low Energy (BLE)}

It is a unilateral and independent communication. On the other hand, Beacons seeking to be found in the environment start transmitting information through the BLE platform at consecutive times. This information can be collected by apps or smartphones, and even Beacons can push or activate the apps. Clearly, BLEs can broadcast information up to 100 meters, which provides a perfect platform for locating people indoors. Throughput may hypothetically reach the limit of $\sim 230 \mathrm{kbps}$, nevertheless authentic applications examined in this evaluation show throughputs limited to $\sim 100$ kbps; the extreme accessible range is strictly reliant on the radio power, and it goes up to a limited tens of meters; the maximum number of nodes in the network depends on connection constraints, on the network architecture and particular device characteristics, however it is typically lower than 10 [17]. BLE beacon networks suffer from the short energy lifetime. This persuades supplementary maintenance also. [18]

To use the BLE in hospital environment, there are factors to be consider of and in below we will show our design for one mid-sized hospital. Figure 6 shows that how the proposed BLE enable sensors and required components have to be interconnected and how is the architecture of the system implemented in mid-size hospital to locate and monitor the patients' location and vital signs activity in real-time.

All public areas must be equipped with the BLE sensors to give us the capability of sending messages and send the navigation aids data to user's cell phone. Environmental sensing BLE sensors must be installed on each room to sense and gather the temperature, humidity and light data. During the treatment period, vital BLE sensors/readers have to be attached to monitoring units and used by patients (like smart watch). Correspondingly, medical equipment link X-Ray and MRI machines must be connected to BLE network to have them monitored and be controlled via Central Point [19]. In all hospitals' ER section is very important and critical and arrived patient must be taken under the monitoring immediately so with attaching the wearable BLE sensors at patient arrival all vital signs will be monitored immediately, and it may help to save a live instant. 


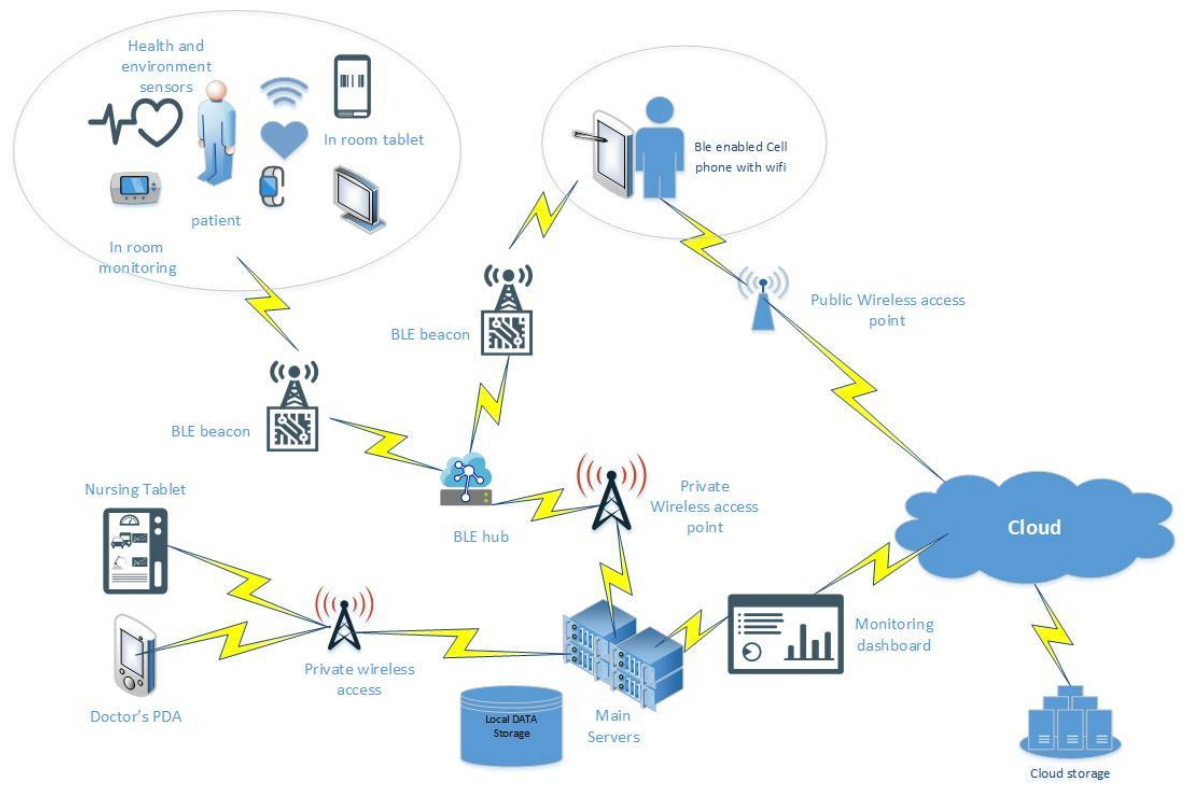

Fig. 6. The proposed BLE based on Beacons model (Total system architecture and components relation)

This modular WSN designed contains BLE wearable and fix sensors to sense the patients' vital signs, sensors to capture and monitor environment conditions such as temperature, humidity and light. All sensors send their data via different kind of media like WiFi, BL/BLE, and WSN to the application-based processing units like doctors' mobile phone and installed in room tablets. For LBS section, we installed BLE iBeacon sensors in public spaces and alleyways. Processed information sent via edge gateways that are installed at the hallways and rooms to the main local servers. Results will be used in nursing stations as a real-time monitoring purpose and also to be shared with other connected hospitals for medical purposes such as drug allergic monitoring or healthcare authorities for performance monitoring. To increase the data availability and security, we store all data in a cloud-based storage and sync it with the local servers.

\section{Implementation}

Using WSN and BLE can help and improve the health care system and hospitals to the server more efficient services and can improve the quality of life. WSN and BLE infrastructure design to use in healthcare environment. The proposed design enables hospitals and healthcare system authorities to have an enriched ecosystem and practical data of patients, disease and quality of service in public health section.

To illustrate the concept, the communication between the Beacons and the BLE platform is discussed. This platform will be distinguished by the following four components: 


\subsection{UUID}

It is a 16-byte string, which is shared by most beacons. If someone is in a hospital and has access to a program or app to take turn and other services, these strings allow the hospital program to figure out the bit string coming from the beacons in the environment; given that the information location and suggestions are sent to the user.

\subsection{Major}

As the iBeacon protocol is used in this work, the detection of Bluetooth Beacon is based on the UUID of the beacon and its MAC Address. Then, by using the Beacons major and minor ID, there will be a capability to distinct location of Beacon groups. The warehouse beacons are also constituted with an explicit major, which is based on the office spaces and using of a major ID for the same. Figure 7 is presenting this ability.

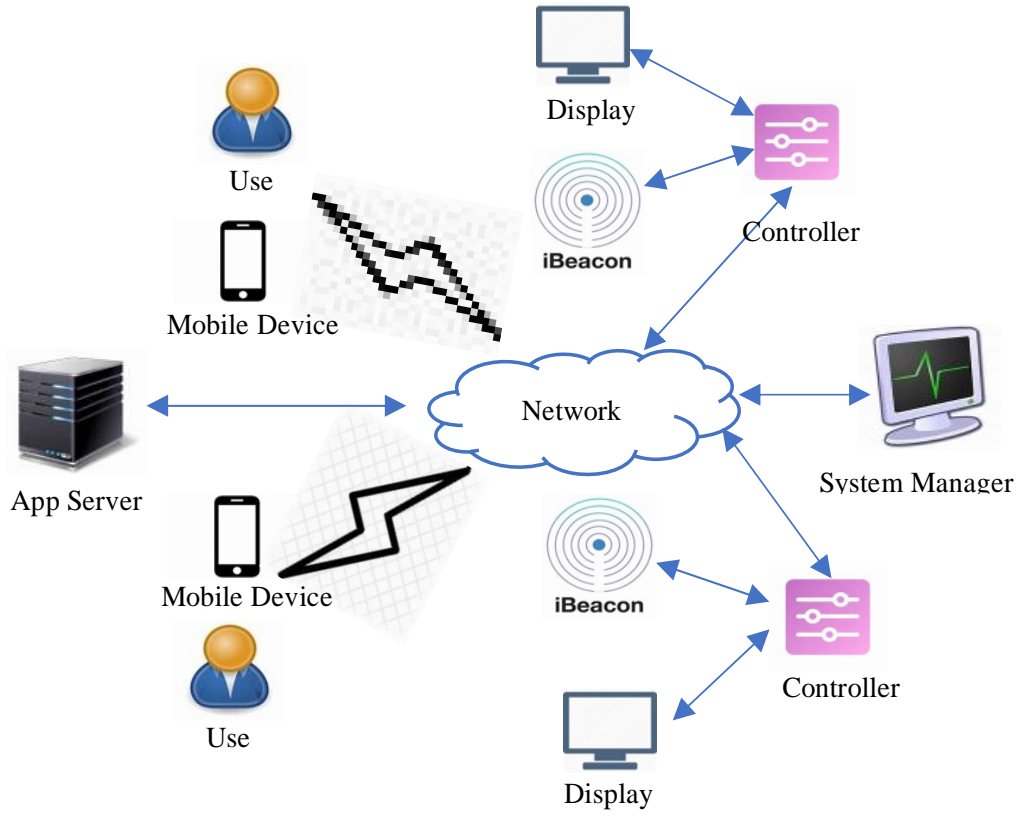

Fig. 7. The way iBeacons communicate with BLE in the proposed model

\subsection{Minor}

A two-byte string with information completely different from each beacon that contains unique information. For example, if someone is at the hospital, the beacon installed at the front door sends a welcome message; however, the beacon near the pharmacy offers unique offers of drug advertising and supplies. 


\subsection{TX Power}

This parameter has the task of measuring the signal strength. The user's exact location is determined by measuring the reciprocal signal strength. The major difference between GPS and Beacon is that the first and foremost differentiation is the data transmission platform, which is satellite in the GPS and Bluetooth in Beacon. This makes the difference between the two technologies in the indoor and outdoor environments.

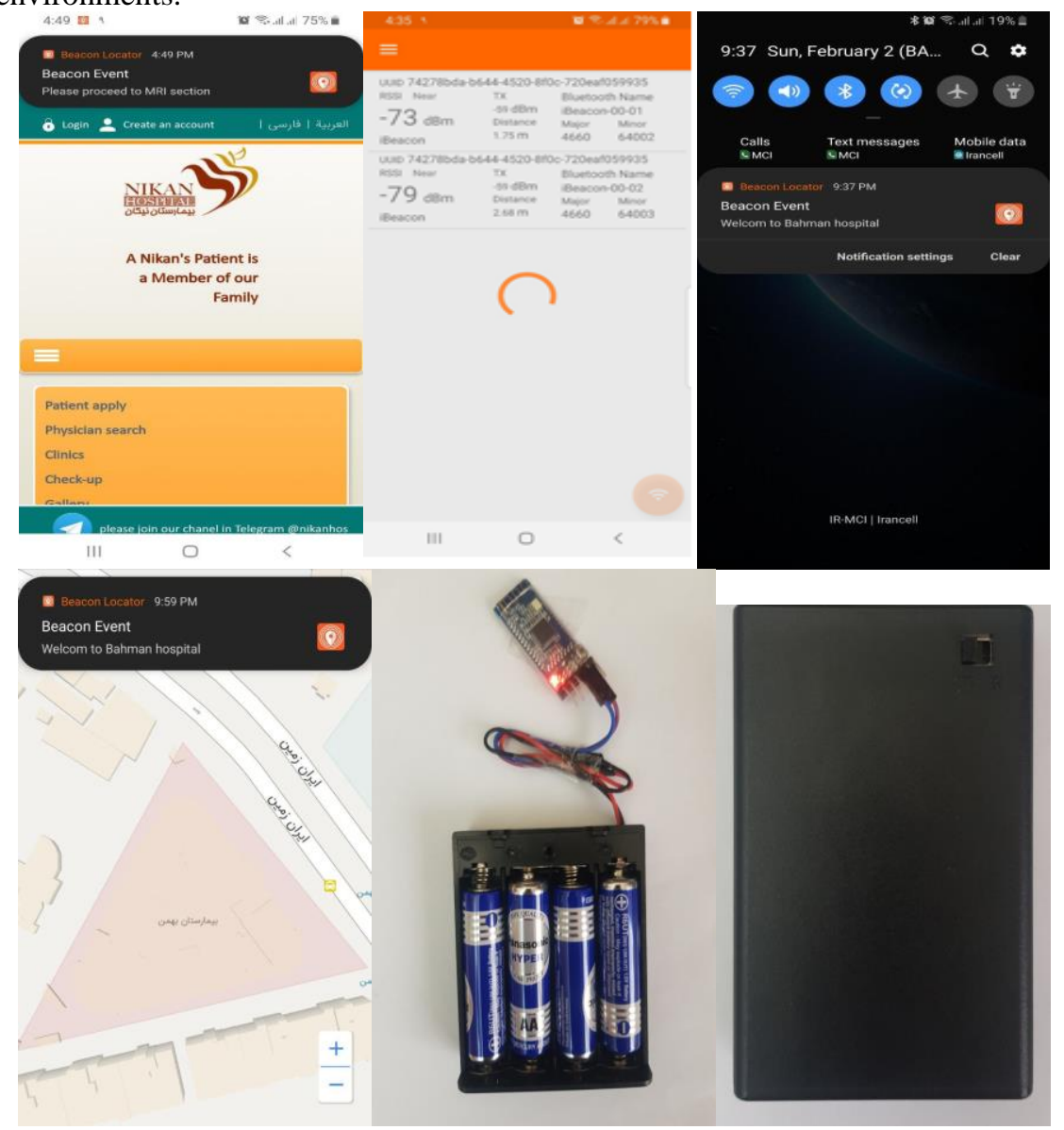

Fig. 8. Designed hardware and software used in mid-size hospital

Now the explained concept is considered in the field of hospital. Assuming that the hospital has different departments such as radiology rooms, doctors' and pharmacy waiting rooms, pediatric dentistry, etc., there is a need for several beacons at each location, each of which is distinguished by UUID (Major\& Minor). Each beacon should be named for a closer look and in larger samples. This 
naming and the resulting information have led to a consensus on the patient information of each ward or unit, which in turn leads to meet the needs.

Designed hardware works under Apple's iBeacon protocol (Figure 8) that broadcast its UUID, Minor and Major to advertise and crowd sensing device use BLE ver4.2 standards.

Prototype hardware and software installed in a mid-size hospital, advertising messages and gathering basic users/environmental data. Figure 9 shows how each beacon can help to manage the information and resource integrity of the hospital. For example, one can refer to the number of times a patient visit's clinic and receive medical attention (Figure 9). A deeper look at this problem allows going farther and addressing people's information more closely. As an illustration, by writing a program, one could achieve a fully documented, personalized history of patients or add how often each patient visit, his waiting time, physician's opinion, type of illness and etc. to other uses of this technique.

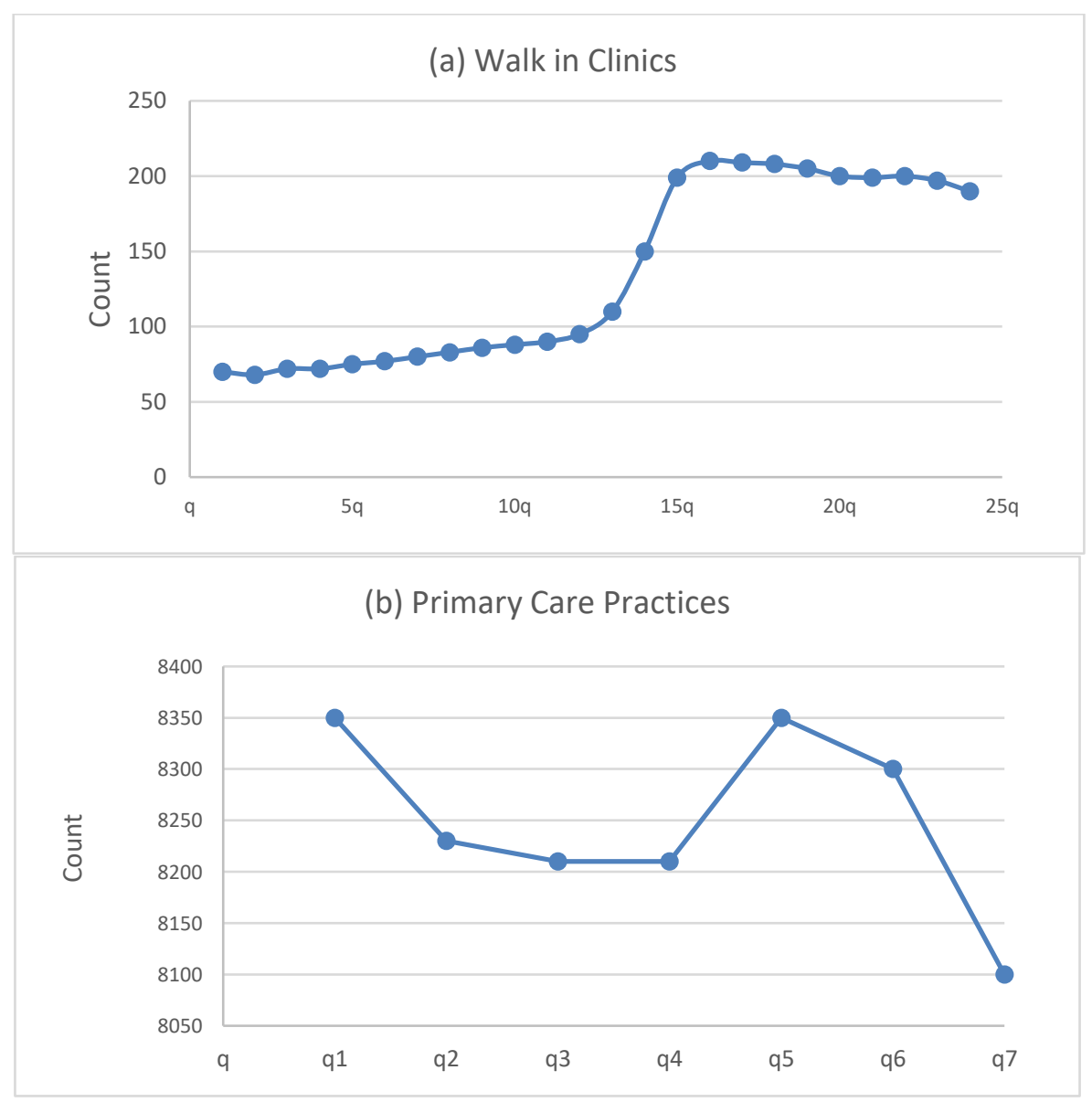

Fig. 9. The Number of times patients visits the clinic and receive medical attention 
All gather data will be stored on local and cloud servers so with this scenario critical data can be available any-time, anywhere, and can be shared between doctors and also other medical staff around the hospital or even another hospital with more doctors and sergeants.

The important part of the system design is the total infrastructure. To achieve the best performance and output of using the BLE, based on its nature and mobility, it is mandatory to design and implement the cloud and fog layer infrastructures. By doing so our real-time data and events will be stored on the cloud-based system via fog layer, and all data will be available for patients and health care system and can be used internal or external (privacy and confidentiality have to be checked by authorities).

To design such an environment, there are several parts to be taking care of: Type of sensors such as body sensors, ingestible sensors, environment control/ monitoring sensors, hence sensors that are connected to the critical machines such as MRI, X-Ray units.

The second design challenge is the data transport media and techniques. The common ways are using wireless access points, cellular network to transmit the data and Bluetooth media gateways. All can be use at the same time to transport the data to the fog layer [20]. Fog layer is the middleware between sensor and sensor gateway's layer and the cloud layer. From fog layer, all data will be interchanged to the cloud layer and will be stored and backed up at the cloud layer. All data can be accessed and used from anywhere anytime. Hospitals, patient, healthcare system and healthcare department authorities will have real-time and archived data via cloud layer.

\section{Results}

By examining the subject, it is concluded that iBeacon technology helps to make a great progress towards the intelligent hospital. This intelligence provides the patients, physicians and diseases information as a valuable source. Using all four components of the BLE technology with the combination of mobile apps, data network based on fog and cloud infrastructure and RSSI navigation control systems, battery enhancement methods, ecosystem can be used in healthcare industry as a key point of monitoring, data gathering and improve the healthcare services.

We installed sensors in ER, MRI and X-ray rooms to achieve the best data from users and find the crowded times of the day. Correspondingly, we installed in each room to monitor the room's environment and its belongings. We attached BLE wrist bands to patient wrist so all vital signs be monitored during the pilot period. All WNS data gathers and stored in a cloud-based storage for further use. Other valuable points of this technique include the fact that there will be a great deal of time savings for patients and physicians.

According to the research done in these articles, it can be concluded that the following results are obtained using the LBS technology:

- Admission automation

- Reduced patients' waiting time

- Reduced registration, dismissal, patient acceptance time manually or using iBeacon technology

- Achieving a database of patient information

- $\quad$ Ease of tracking for patients 
- Coordination of the medical team (informing the staff of the work plan, detailed planning of activities, complete information on the medical conditions of each patient)

- Accurate and reliable reporting of human resources and occupied space (presence of staff in specific areas, specific patients' presence and tracking of, etc.)

- Smart emergency support (safety measures and evacuation relief in public areas or hospital buildings)

This scenario can be implemented in any size of hospital and even in small clinics and can improve the service level and also gather important information about the staff, doctors, and environment condition and with this kind of information owners can monitor the data, as well, governments and healthcare system authorities can have a better point of view about the healthcare ecosystem.

\section{Conclusions and Future work}

By implementing the BLE technology in hospitals, we can organize the potential of providing useful applications to the healthcare industry that can be trusted and relay on by medical staff and patients. BLE enabled devices are being used by many people in form of smart watches and wearable devises also BLE enabled medical units and machines are available in hospitals/small clinics aiming to serve better healthcare services and achieve accuracy and flawless action in administration of the hospital as well as the medical service provided to the patients.

By using BLE in healthcare environment, the vast amount of data will be generated. These data contain patient's vital data, environments data healthcare system's data. For future works it's suggested to focus on data analytics and data mining to use all capability of the gathered data via using the BLE.

\section{References}

[1] Pinchbeck EW. Convenient primary care and emergency hospital utilisation. Journal of Health Economics. 2019; 68: p. 102242.

[2] Yu B, Xu L, Li Y, editors. Bluetooth Low Energy (BLE) based mobile electrocardiogram monitoring system. 2012 IEEE International Conference on Information and Automation; 2012: IEEE.

[3] Alam MM, Ben Arbia D, Ben Hamida E. Wearable Wireless Sensor Networks for Emergency Response in Public Safety Networks. In: Câmara D, Nikaein N, editors. Wireless Public Safety Networks 2: Elsevier; 2016. p. 63-94.

[4] Frisby BN. Capitalizing on the inevitable: Adapting to mobile technology in the basic communication course. Basic Communication Course Annual. 2017; 29(1): p. 8.

[5] Buhalis D, Leung R. Smart hospitality-Interconnectivity and interoperability towards an ecosystem. International Journal of Hospitality Management. 2018; 71: p. 41-50. 
[6] Salam HA, Khan BM. Use of wireless system in healthcare for developing countries. Digital Communications and Networks. 2016; 2(1): p. 35-46.

[7] Nowotarski P, Pasławski J, Mielcarek D. Accuracy of BLE systems in the H\&S improvement aspects in construction. Procedia Engineering. 2017; 208: p. 98-105.

[8] Li Z, Yang Y, Pahlavan K, editors. Using iBeacon for newborns localization in hospitals. $10^{\text {th }}$ International Symposium on Medical Information and Communication Technology (ISMICT); 2016: IEEE.

[9] Gershoni D, Nosrati FD. Smart personal emergency response systems (SPERS). Google Patents; 2019.

[10] Lin Y-W, Lin C-Y. An Interactive Real-Time Locating System Based on Bluetooth LowEnergy Beacon Network $\uparrow$. Sensors. 2018; 18: p. 1637.

[11] Yaeghoobi KS, Soni M, Tyagi S. Schedule communication routing approach to maximize energy efficiency in wireless body sensor networks. Smart Structures Systems. 2018; 21(2): p. 225-234.

[12] Rahmani AM, Gia TN, Negash B, Anzanpour A, Azimi I, Jiang M, et al. Exploiting smart eHealth gateways at the edge of healthcare Internet-of-Things: A fog computing approach. Future Generation Computer Systems. 2018; 78: p. 641-658.

[13] Baek S-K, Chanho Y, Kim E, Cha JS, Chang SC, Ko YJ. Method and apparatus for transmitting and receiving reference signal using unlicensed band. Google Patents; 2019.

[14] Jaiswal K, Sobhanayak S, Turuk AK, Bibhudatta SL, Mohanta BK, Jena D. An IoT-Cloud Based Smart Healthcare Monitoring System Using Container Based Virtual Environment in Edge Device. International Conference on Emerging Trends and Innovations In Engineering And Technological Research (ICETIETR)2018. p. 1-7.

[15] Surian D, Nguyen DQ, Kennedy G, Johnson M, Coiera E, Dunn AG. Characterizing Twitter discussions about HPV vaccines using topic modeling and community detection. Journal of medical Internet research. 2016; 18(8): p. e232.

[16] Gautam PR, Kumar S, Verma A, Rashid T, Kumar A. Energy-Efficient Localization of Sensor Nodes in WSNs Using Beacons From Rotating Directional Antenna. IEEE Transactions on Industrial Informatics. 2019; 15(11): p. 5827-5836.

[17] Tosi J, Taffoni F, Santacatterina M, Sannino R, Formica D. Performance evaluation of Bluetooth low energy: a systematic review. Sensors. 2017; 17(12): p. 2898.

[18] Jeon KE, She J. User Existence-aware BLE Beacon Firmware for Extended Battery Lifetime. 2019.

[19] Surian D, Kim V, Menon R, Dunn AG, Sintchenko V, Coiera E. Tracking a moving user in indoor environments using Bluetooth low energy beacons. Journal of Biomedical Informatics. 2019; 98: p. 103288.

[20] Farahani B, Barzegari M, Shams Aliee F, Shaik KA. Towards collaborative intelligent IoT eHealth: From device to fog, and cloud. Microprocessors and Microsystems. 2020; 72. 